\title{
¿SIRVE PARA ALGO DECENTE LA FILOSOFÍA POLÍTICA?
}

\author{
Félix Ovejero Lucas \\ Universidad de Barcelona
}

RESUMEN. ¿Podemos esperar alguna ayuda de la filosofía política para el juicio político? En la obra de Garzón Valdés hay razones para dar una respuesta positiva a esa pregunta. Particularmente en su propuesta de un "criterio de irracionabilidad» que «funcionaría de manera similar a la falsabilidad en las ciencias naturales», sirviendo de limite a lo «meramente racional». El problema es si la vía de los consensos universales suministra criterios con real contenido informativo. Desde otro punto de vista, al final nos encontramos con el problema de siempre, que impide sostener la comparación: en filosofía práctica no hay posibilidad de control empírico. Pero disponemos de otros tipos de controles, más inseguros pero no irrelevantess. Hay uno que Ernesto supera con la mejor nota: la veracidad en el trato con las propias ideas.

Palabras clave: Ernesto Garzón Valdés, filosofía política.

ABSTRACT. Can we expect any help from political philosophy for the political judgment? In the work of Garzón Valdés there are reasons to give a positive answer to that question. Particularly in its proposal of a "criterion of irrationability» that «would work of way similar to the falsability in natural sciences", providing limits to the «purely rational». The problem is if the route of the universal consensuses provides criteria with real informative content. From another point of view, in the end we find the enduring problem, which prevents to maintain the comparison: in practical philosophy there is no possibility of empirical control. But we have other types of controls, more uncertain but relevant. There is one that Garzón Valdés passes with the best grade: truthfulness in dealing with his own ideas.

Keywords: Ernesto Garzón Valdés, political philosophy. 


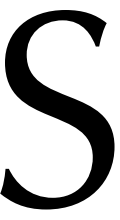

e aprende mucho en las librerías de los aeropuertos. En los últimos años abundan los libros de filosofía práctica. No siempre se trata, o a mí no me lo parece, de «filosofía barata», de manuales de autoayuda, no al menos en el sentido en el que entre nosotros, gente del oficio, hablamos de «manuales de autoayuda», ya saben, con aire displicente, como sobrados de talentos en la gestión de nuestros menesteres terrenales. Por lo general, son libros que recuperan una de las concepciones más dignas del quehacer filosófico: proporcionar una suerte de ingeniería del alma con la que enfrentarse al enojoso oficio de vivir, al arte de ordenar cabal - y felizmente - la vida de cada cual y, por ende, si la tarea se realiza como Dios manda, la vida de todos. En fin, los asuntos que entretenían a los estoicos o a ARISTÓTELES, a quienes, como los primeros, sabedores de lo incierto del mundo, limitaban sus investigaciones a las terapias del deseo y la voluntad, y a otros más optimistas, como el estagirita, confiados de que también en la vida compartida había razones para la esperanza.

Seguramente el lector de esos libros pensará que los filósofos somos gente justa y noble, de vida ordenada y juicio ecuánime, ocupados en alcanzar un trato sereno con nuestros negocios y amores y en resolver los problemas reales de la ciudadanía. Así que, como nunca sobran los amigos ni los admiradores, guardemos el secreto. Que no se nos cuele en ningún congreso y descubra la naturaleza del negocio. Porque si se nos cuela, no tardará en perdernos el respeto, en pensar que lo nuestro son locuras sin fundamento. Vería que nos entretenemos en discutir cosas bastante extrañas: que si existe el rey calvo de Francia, que qué se siente al ser un murciélago o un cerebro en una cubeta e incluso asuntos propios de Star Treck (tierras gemelas, teletransportación, etc.). En fin, como se ve, cuestiones de mucho provecho para la mejora del mundo y de nuestros espíritus.

Como no hay que hacer trampas ni hablar a medias, y estoy entre gentes en condiciones de dejar en ridículo al Cardenal Castrillón Hoyos, capaz de dividir un cabello en 17 partes iguales, el matiz y la cautela se imponen inmediatamente. Sí, es cierto que las ideas se pulen en los casos extremos, que sin ayuda de los contrafácticos es difícil hacer teoría moral, que los experimentos mentales son fundamentales incluso en las teorías físicas más importantes. Todas esas razones son atendibles, por más que no falten las idas y las venidas. Hasta se podrían añadir algunas consideraciones adicionales, algunas solemnes, como que constituye una capacidad propia de la especie humana, en virtud de su potencial lingüístico, la posibilidad de escapar a sus constricciones preceptuales y de explorar conjeturalmente mundos distintos de su ámbito de experiencias, y otras más circunstanciales, como el hecho de que no pocos de los filósofos con más disposición a enlodarse en los asuntos colectivos (la política de Bush, la globalización, por no mencionar el multiculturalismo) se manejan como la elegancia del elefante en la cacharrería en lo que atañe a la pulcritud argumental.

Bien, admitamos que los filósofos nos desenvolvemos con enorme soltura y fineza en bastantes mundos posibles, que, si de pronto, hay que empujar a un gordito para 
desviar la trayectoria de un tranvía desbocado y salvar a unas cuantas gentes, mientras las personas normales empezarían a temblar en un mar de dudas, nosotros actuaríamos con resolución clarividente y serena, con el temple de astronauta en apuros. Pero, también es obligado admitir que casos como éstos no se dan cada día, que las decisiones en las que esperamos que los filósofos —y en particular los filósofos políticos- nos iluminen son más cercanas. Y, en todo caso, cabría pensar que, puesto que mostramos tanta brillantez en las situaciones más imprevisibles, también deberíamos ser capaces de proporcionar pautas para encarar «los comunes casos de toda suerte humana», que diría Borges. Sería de esperar, al fin, que la persona capaz de deslindar entre cuarenta ideas de igualdad, respeto o responsabilidad, además de no ser un imbécil moral o un miserable mal bicho, opine con algún criterio sobre los debates políticos de cada día. Vamos, que confiamos que el Doctor House, además de las enfermedades excepcionales, nos cure un triste achaque.

Para ser sincero, no estoy seguro de que sea así. Al menos a mí me dan que pensar, y no para bien, las opiniones vertidas — y el verbo es muy ajustado - por bastantes académicos norteamericanos a propósito del 11-S: en el mejor de los casos, trivialidades campanudas; en el peor, himnos patrióticos. Puestos a contarlo todo y no pescar sólo en mares ajenos, aquí, entre el fuego amigo, quizá no esté de más acordarnos de los elogios de un reputado filósofo republicano irlandés a la gestión política de nuestro presidente: puros palos de ciego.

Hace apenas cuatro años, Frederic SPOTTS, en su libro Hitler and the Power of Aesthetics, nos confirmó que la más refinada sensibilidad estética puede convivir sin rozaduras con el delirio moral. Resulta difícil pensar que algo parecido pudiera suceder con la filosofía moral, dada la propia naturaleza del empeño: ordenar la vida práctica. Pero lo cierto es que no faltan los ejemplos que le hacen pensar a uno que a nuestros azacaneos le cuadra impecablemente aquella rotunda sentencia de Unamuno a propósito del ajedrez: «El ajedrez es un juego que desarrolla la inteligencia... para jugar ajedrez». Pues eso, a veces pareciera que la filosofía moral desarrolla la sensibilidad moral... para seguir jugando a la filosofía moral. Poco más.

\section{¿LA FILOSOFÍA O LOS FILÓSOFOS?}

Quiero precisar que es un problema de disciplina, antes que de personas, de la debilidad de la disciplina. El problema no es si los filósofos políticos somos gente deshonesta o de pocas luces sino si la filosofía política es capaz de proporcionarnos base racional para la política. Es muy posible que el estudioso de la mecánica de fluidos sea un inútil desmadejado a la hora de reparar una cañería o de conducir un coche pero, desde luego, su disciplina es de suma utilidad para resolver los problemas de la fontanería y del tráfico. Entre nosotros no parecen ser así las cosas, y ahí tenemos a Jeremy WALDRON, por cierto, en bandería con NOZICK, sosteniendo que hay poco que esperar de la reflexión teórica. Si llamamos a las cosas por su nombre, en román paladino, para WALDRON la filosofía política pertenece, con el debido respeto, al género de las pajas mentales. Con el debido respecto, naturalmente, a dicho género. Justo es decir que, terciando en el mismo debate, Martha Nussbaum, siempre pertrechada con sus inevitables griegos, ha proporcionado apreciables razones para suscribir la divisa aristotélica 
de que el fin de la reflexión política «no es el conocimiento, sino la acción» (EN, 1095a, 5 ), de que «no investigamos para saber qué es la virtud, sino para ser buenos, ya que en otro caso sería completamente inútil» (EN, 1103b, 25).

Hecha la advertencia acerca de que el problema es de la disciplina, no quiero dejar pasar la ocasión de decir que, si tantas veces los problemas de la disciplina se resuelven en problemas de personas (en lugar de «qué se sigue — si se sigue algo- de la teoría de la acción comunicativa sobre esto» la pregunta es «pero, qué piensa HABERMAS sobre esto», duda que, por cierto, siempre se podría resolver teniendo un teléfono a mano), es porque la disciplina se muestra insegura y con criterios de tasación equívocos. La flaqueza de las teorías otorga un papel desmedido a unas opiniones políticas, formuladas por lo general a bote pronto, que no siempre guardan relación con las reflexiones profesionales. Si las conjeturas fueran sólidas, todo sería más sencillo. Al menos, el filósofo resultaría menos protagonista. Son estos caminos que no quiero recorrer ahora, aunque algo me gustaría decir más tarde, al final de mi charla.

En fin, recupero el hilo y perfilo el problema. ¿Podemos esperar alguna ayuda de la filosofía política para el juicio político? Creo que en la obra de Ernesto hay razones para dar una respuesta positiva a esa pregunta. Una opinión, en contra de lo que podríamos esperar, bastante infrecuente. Se conoce que somos personas dadas al funebrismo porque la mayor parte de los filósofos políticos parecen convencidos de cultivar una disciplina inútil. Algo que, admito, no deja de producirme perplejidad, porque, si ese es el caso, entonces todos a territorios más firmes y controlables, a la lógica y al análisis duro. O sin más, a la literatura; al dilema del Ortega joven: «o se hace literatura o se hace precisión o se calla uno».

\section{TRES (MODERADOS) PESIMISMOS}

Tengo inventariados bastantes pesimismos. Examinaré algunos de ellos, antes de ocuparme de lo que entiendo es el punto de vista de Ernesto. En todo caso, dada la naturaleza festiva de esta reunión y como no quiero frustrar incipientes vocaciones, sólo mencionaré tres de los más tibios. En realidad, no es nieguen las posibilidades prácticas de la filosofía política, sino que se muestran escépticos acerca de si podemos llegar a descubrirlas. Son tres perspectivas diferentes que, a su vez, arraciman diversas tesis que no estoy del todo seguro resulten compatibles entre sí. Así que, advierto, voy a forzar un poco el trazo.

- Una primera respuesta a nuestra pregunta, una respuesta institucionalmente pesimista, nos vendría a decir que las teorías normativas no tienen ocasión de cristalizar en acciones políticas. Acaso sean buenas, pero da lo mismo porque no está en nuestra mano sacar provecho de su potencial. Ocurre en muchas circunstancias de la vida, en la de cada cual y en la de todos: conocemos el diagnóstico y la solución pero no disponemos de poder - y eso puede incluir nuestra voluntad — para hacer lo que debemos hacer. Por lo que sea, no estamos en condiciones de llevar a cabo las acciones. «Por lo que sea» se refiere casi siempre, como digo, a falta de poder. Que no acabemos con las hambrunas o la pobreza absoluta no es un problema de la teoría, de falta de propuestas sensatas y bien fundadas, sino del mundo. Conviene no olvidar estas cosas para no 
seguir llamando a la puerta equivocada, para no achacar otras culpas a las teorías que las que estrictamente les corresponden.

Pero no siempre es un problema de poder. Otras veces, y es el caso que más nos debería interesar, el problema es de nuestras instituciones políticas, empezando por nuestros sistemas democráticos, que se muestran insensible a la información relevante, que dificultan - por no decir que hacen imposible - el triunfo de las propuestas claras y distintas. La resabiada coletilla de «las elecciones se ganan en el centro» es la versión manoseada periodísticamente de resultados poderosos de la teoría de la elección racional: el teorema del votante medio o en otra formulación, el teorema de Hotteling. En una interpretación rústica y sociológica, esto es, doblemente rústica, tales resultados vendrían a decir que, en nuestras sociedades, sin agudas polarizaciones de clases, para llegar al poder, para conseguir la mayoría, hay que limar aristas a las ideas, desdibujar las propuestas, escamotear los problemas, contentar a todos, esto es, no decir nada. Al final, las organizaciones políticas se ubican en un complicado dilema entre la claridad y el poder que, para lo que nos interesa, quiere decir que no hay modo de hacer uso de las tecnologías que las teorías normativas nos pudieran proporcionar. No creo que sea casual que entre los políticos cada vez se extienda a más asuntos y más fundamentales esa enojosa descalificación, bastante insidiosa, de hacer «electoralismo con los asuntos importantes» (terrorismo, emigración, modelos energéticos, pensiones, etc.). Nos vienen a decir: con las cosas de comer, no se juega, o al menos, no en el debate democrático. Hay, para decirlo con Ernesto, un coto vedado al coto vedado, una dificultad para que los asuntos serios puedan ser objeto del debate político.

Es tarea de la ciencia política, empírica y analítica, desentrañar los entresijos e implicaciones —nada desdeñables - de este mal llevar entre la democracia y los problemas importantes. Aquí tan sólo me interesa destacar la existencia de un razonable punto de vista que sostiene que, aunque la filosofía política nos proporcione guías de acción, las proporcione o no, no tenemos un modo seguro de saberlo. Un juicio que pueden compartir tanto los escépticos acerca de la bondad de la teoría como los escépticos acerca de la bondad del mundo. Y, por supuesto, todos aquellos que creen que la política en serio, es poder, que las buenas razones están bien para decorar y dar relumbrón a los discursos pero poco más.

- Un segundo pesimismo sostiene que no hay modo de sacar provecho de los desarrollos normativos porque lo que falla es el acompañamiento, la base informativa sobre la que cimentar las intervenciones. Pudiera suceder que, por ejemplo, llegásemos a la conclusión de que es justo distribuir los logros de la sociedad atendiendo a las necesidades de las gentes, pero que no hubiera manera de hacerlo porque no tenemos un modo fiable de conocer tales necesidades. Pero no me quiero referir a ese tipo de problemas, interesantes, pero que caen más en el negociado de los economistas que en el de los filósofos, sino a otros que han servido —en sus diversas formas- para condenar las intervenciones sociales, sobre todo las radicales, las ingenierías sociales. En otro lugar, he agrupado estos puntos de vista bajo el rótulo un tanto imponente de «críticas epistémicas de las intervenciones políticas». Por lo general, arrancan, con buen tino, estableciendo un trazo entre las acciones individuales, y las políticas, las colectivas. Las primeras no presentarían mayores problemas. Mal que bien, en la gestión de nuestras vidas, una vez aclarados los propósitos, podemos disponer de una pauta elemental acer- 
ca de cómo llevarlos a cabo. La teoría matemática de la decisión y la teoría de juegos nos proporcionan reglas bastante fiables de cómo hacer las cosas, incluso en aquellas situaciones en donde no andamos muy sobrados de información acerca del mundo: recabar las informaciones relevantes sobre las alternativas disponibles, incluidas las probabilidades, jerarquizar las preferencias y actuar de modo coherente con unas y con otras. Entre filósofos a esas reglas, en otros tiempos, no sin jactancia faltona, se las llamaba «racionalidad instrumental». En la práctica, nos permiten gestionar una parte importante de nuestros afanes, entre los que no se incluyen, cierto es, los que tienen que ver con la felicidad, que se escriben con renglones torcidos, que hay que abordarlos de soslayo, como sin querer. Pero con esa notable excepción, y alguna otra, menor, sirven.

Las cosas son bien distintas cuando se trata de la casa común, de los asuntos de la filosofía política. Incluso si podemos poner nombre a los anhelos colectivos, y la economía del bienestar - ARROW mediante- nos ha recordado que no es una tarea baladí, no habría modo, dirán los pesimistas epistémicos, de disponer de una buena cartografía de navegación, sea porque no hay — ni puede haber- teorías sociales disponibles, al menos en lo que atañe a las intervenciones de gran calado, sea porque, incluso si disponemos de tales teorías, la más ínfima variación en las condiciones iniciales nos enfila en veredas imprevisibles, o, con más gracia, con el Borges de El Sur: «ciego las culpas, el destino suele ser despiadado con las menores distracciones». Vamos, que como no hay modo de poder decir «nos vamos a la guerra de los treinta años» no cabe planificar las batallas. POPPER y el efecto mariposa, para decirlo pronto, y seguramente, mal. Repárese, en todo caso, que tampoco ahora el problema está en el territorio de la teoría normativa.

- El último de los pesimismos que quiero hoy mencionar apunta directamente al corazón de la filosofía política. Sus abstractas prescripciones serían un terreno poco firme en donde plantar pie para afincar decisiones políticas que requieren «el análisis concreto de la situación concreta», como decía el protagonista de la revolución rusa. El problema sería de principio: no es que nuestras herramientas analíticas actuales puedan ser susceptibles de mejora o cambio, por ejemplo, con una revolución metodológica o teórica. La política es acción práctica, trata con lo particular, con el mundo, no con descripciones del mundo como las teorías. Sencillamente, no podemos esperar exactitud donde no cabe y, en las decisiones prácticas, políticas, no cabe. Creer algo distinto será simple y llana estulticia, nos dirá ARISTÓTELES: «es propio del hombre instruido buscar exactitud en cada materia en la medida que lo permite la naturaleza del asunto; evidentemente, tan absurdo sería aceptar que un matemático empleara la persuasión como exigir de un retórico demostraciones» (EN, 1094b, 25). Las teorías normativas vendrían a dotarnos de una ilusión de precisión que confunde más que ayuda.

En ese pesimismo coinciden diversas estrategias de argumentación. Las de raíz aristotélica aúnan la tesis epistemológica de que no hay conocimiento de lo particular con la convicción de que la política es, finalmente, práctica, acción sobre la realidad, no con informes sobre la realidad. En palabras del propio ARISTÓTELES: «(La prudencia, la Phronesis) es práctica y la acción tiene que ver con lo particular» (EN, 1141b, 24). Así las cosas, no habría posibilidad de hacer uso político de las teorías normativas, abstractas por naturaleza. En el mejor de los casos, cabría recalar en un saber prudencial, capaz de ponderar las circunstancias y los valores comprometidos en las decisiones; en el peor, en una suerte de genialismo que atribuye a ciertos individuos — cuando se los 
quiere decorar se los llama hombres de estado — una supuesta sensibilidad especial para los quehaceres políticos, una sensibilidad pareja a la del artista. Otra veta, de inspiración hegeliano-marxista, llegará al mismo pesimismo pero discrepando de la premisa epistémica: que no tengamos ciencia de lo particular no nos impide alcanzar un conocimiento de lo particular sobre el que basar la práctica política. Ese conocimiento, dialéctico, no muy diferente del que cuaja en la mejor historiografía, sería el resultado de una composición de las abstracciones de las distintas ciencias: «una síntesis de multiplicidad de determinaciones», según la conocida formulación de MARX en su Introducción a la crítica de la Economía Política (1857). Sea como sea, ninguna de las dos estrategias, ni la que apela a la capacidad ponderadora del sabio o del genio, ni la que anda en pos de un conocimiento totalizador, sintetizador de las diversas abstracciones de la ciencia, deja en buen lugar a las teorías normativas. En un caso o en otro, estamos lejos de un conocimiento susceptible de ser reglado. Es antes arte o artesanía que algoritmo.

Los puntos de vista expuestos hasta aquí —y son los más contenidos- invitan a esperar poco de la filosofía política. En realidad, si las cosas fueran de ese modo, lo más honesto sería levantarnos ahora mismo y dirigirnos a la oficina de empleo más próxima, confiando en que, sin preguntarnos por nuestro currículo, nos ofrezcan algún trabajo en el que no podamos perjudicar mucho a la humanidad. Afortunadamente, algunos, no muchos no comparten tan sombrío diagnostico. Ya he mencionado a la NUSSBAUM en su polémica con WALDRON y, por supuesto, siempre hay que acordarse de los Ilustrados, quienes, en su mayoría, compartían el diagnóstico, el emplazamiento, que tan claramente trazó uno de ellos, HeLveTius: «si no se puede fundir con la política, la moral es una ciencia frívola» (De l'esprit, 2, 15, 161).

\section{AL MENOS, LO QUE NO DEBE SER}

Ernesto es uno de esos pocos que creen que la filosofía política puede servir, además de para reunirse con los amigos y orearse un poco, para ayudarnos en el juicio político. A pesar de que, entre bromas y veras, con su característica bonhomía, nos dice que carece de talento para escribir en las distancias cortas, como hacemos algunos que de vez en vez nos contaminamos como gacetilleros, caben pocas dudas de su interés por la política. De que cree que alguna responsabilidades ha dejado testimonio, negro sobre blanco, entre otros lugares, en una reflexión sobre la fundamentación racional de la praxis política, a partir de la obra del filósofo peruano MIRÓ QUESADA, donde sostenía con cierta gravedad que «la misión del intelectual es desenmascarar los mitos que encubren la injusticia y la arbitrariedad de la sociedad que le ha tocado vivir». Nada sorprendente. Hay sobrada evidencia de que no se afilió a los héroes o modas del momento y, lo que no es menos importante, de que perdió o dejó pasar oportunidades que exigían el peaje de las convicciones. Con el léxico austero y sombrío de los economistas: ha tomado decisiones que tienen un alto coste de oportunidad. Por supuesto, eso sólo sucede cuando las intervenciones no son a favor de corriente. Cuando digo que «no son favor de corriente» no me dejo llevar por la inercia de las palabras gastadas, no adopto un punto de vista urgentemente ideológico. Creo que no está de más recordarlo en un país en el que en algunos lugares resulta mucho más costoso defender la constitución que condenar a Bush y al sistema, que por supuesto es lo debido. En realidad, 
en último caso, si insistes un poco en la disposición contra el sistema, hasta puede que te caiga un cargo público. Sobre todo si «el sistema» contra el que se rebelan está muy lejos.

Con todo, que Ernesto se haya interesado por la política no supone necesariamente que tenga confianza en los beneficios de la teoría normativa para la política. Quizá suceda que, yo plural y de una sola sombra, para acudir por última vez a Borges, el Ernesto ciudadano se enoje con el mundo y golpee la mesa sin molestarse en consultar al Ernesto académico. Quizá, mira por donde, al fin encontremos un punto de acuerdo entre WALDRON y Ernesto.

Pero me temo que no es así, que tampoco esta vez, qué le vamos a hacer, hay coincidencias, que Ernesto espera más de las reflexiones analíticas que el autor de Derecho $y$ desacuerdo. Seguramente no hay mayor ejemplo que su «coto vedado», una teoría de la que se puede decir cualquier cosa menos que es paniaguada. Yo no me voy a referir a ese desarrollo sino a otro muy emparentado con él, en realidad, a mi parecer, es su soporte último: la propuesta de un «criterio de irracionabilidad» que «funcionaría de manera similar a la falsabilidad en las ciencias naturales», sirviendo de límite a lo «meramente racional». Una propuesta que, «pretende poner freno a lo que podría llamarse "actitud imperialista de las ciencias naturales" y a su presuntuosa actitud de negar la posibilidad de conocimiento en aquellos campos donde su metodología resulta epistemológicamente inaceptable».

La propuesta me parece muy sugerente. Me interesa sobre todo la comparación con la ciencia empírica sobre la que algo quiero decir. Vayan por delante algunas acotaciones menores, que pueden parecer - y acaso lo sean - pejigueras de filósofo de la ciencia, sobre lo que Ernesto llama «las pretensiones imperialistas de las ciencias naturales», sobre la mercancía que se querría exportar, el método de las ciencias naturales, y sobre los exportadores, los científicos. Primero sobre la mercancía: resulta más que discutible que exista nada parecido a una metodología de las ciencias naturales, común y exitosa, que estemos en condiciones de llevar de aquí para allá. El Origen de las especies no se parece en nada a los Principia de NEWTON, por no referirse a cualquiera de los trabajos de EINSTEIN que revolucionaron a principios del siglo pasado varias áreas de la física. Mientras las leyes de la física se exponen en pocas páginas vigorosamente demostrativas el libro de DARWIN es una investigación narrativa, persuasiva, casi novelera, en donde, a lo largo de un montón de páginas, los ejemplos, bien traídos, arropan unas pocas ideas, no siempre claras, a las que sólo exageradamente podemos calificar como teorías. La segunda reserva apunta a los traficantes, a quienes participan de «la actitud de negar la posibilidad de conocimiento» en nuestros negocios, en su parecer, los científicos naturales. Creo que yerra el tiro. Tengo para mí que esa actitud, si se da, es más cosa de filósofos de la ciencia - de los de hace bastante tiempo, también en su arrogancia- que de los científicos, quienes, en los últimos tiempos, no es que rechacen la posibilidad de conocimiento en nuestros asuntos, sino que hay que embridarlos en su optimismo - en otro sentido-imperial, o por los menos, rebajarles el alcance de sus intervenciones naturalistas, no pocas veces triviales y casi siempre puramente conjeturales, sin resultados contables, como sucede con bastantes — que no todosdesarrollos de la psicología evolutiva. Bien pensado, hay una cierta paradoja en la formulación de Ernesto, pues si por una parte, descalifica la actitud imperialista de las 
ciencias naturales, por otro, la describe con escasa vocación colonizadora, en tanto «negaría la posibilidad del conocimiento» en nuestros afanes.

Como digo, ésas son reservas menores. Si las traigo a colación es porque no creo que Ernesto debiera mostrar cautelas respecto a lo que llama programa «imperialista de las ciencias naturales» cuando precisamente, a mi parecer, uno de los puntos de más interés del criterio de irracionalidad es el paralelismo - que él mismo enfatiza- con el tan denostado, y tan sensato por demás, criterio de falsación, una de las escasas propuestas que dota de algún sentido a la fatigosa —e inútil- disputa acerca de la unidad metodológica de las ciencias. Dicho sea de paso: después de tanta de majadería postmoderna uno no puede sino lamentar que POPPER no señoree — por no decir tiranicela filosofía de las ciencias sociales, como sucedió en otro tiempo.

Como es conocido, el filósofo austriaco nos recordó que, en realidad, la ciencia no busca maximizar la verdad. Ir acumulando verdades es la cosa más sencilla del mundo, como bien saben esos concienzudos artistas de la vaciedad que son los componedores de horóscopos. Lo que buscamos son las teorías improbables, las que excluyen más mundos posibles. Por eso nos parece más interesante la afirmación «la velocidad de la luz es de 300.000 kilómetros por segundo» que la de que «la luz va bastante deprisa». Mal que bien, el criterio de falsación nos sirve - bien es verdad que en ejemplos bastante domésticos- para calibrar el contenido informativo de las teorías y de ese modo ayudar a reconocer a las mejores.

La pregunta es si podemos apurar la comparación de Ernesto y sopesar las teorías normativas con su criterio de corrección como hacemos con las empíricas con el criterio de falsación para, en un segundo paso, pertrechados de las más interesantes echarle cuentas al mundo, que es lo que importa. Desde otro punto de vista, algo de eso ha sugerido Amartya SEN, afín en tantas cosas a un autor como DASGUSPTA, tan justamente apreciado por Ernesto. En distintos sitios el premio Nóbel de economía ha sostenido que las teorías normativas operan como filtros informativos. Una teoría que da todo por bueno vendría a ser el equivalente de una tautología, compatible con todo y, por ende, de nulo interés. La afirmación de astrólogo «a usted le sucederá algo» tendría su paralelo en una teoría que sólo nos permitiera condenar la esclavitud.

Pero no sería del todo justo, y aquí viene mi pequeña objeción, si no señalara que la interpretación anterior, epistemológica, del criterio de irracionalidad coexiste con otra más estrictamente normativa, según la cual el criterio de irracionabilidad opera como «un consenso universal acerca del mal radical». En este caso, lo excluido serían «estados de cosas cuyo rechazo sería unánime, independientemente de la concepción de lo bueno que se tenga». No estoy seguro que las dos aproximaciones al criterio de irracionabilidad, la epistémica, que apostaría por teorías poderosas, y la normativa, que busca acuerdos amplios, convivan sin problemas. Que hay puntos de solapamiento, seguro, pero también de tensión. El mayor consenso universal lo tienen las tautologías, las que menos contenido informativo proporcionan, las que nos dotan de menores criterios políticos.

Mis simpatías se decantan por la interpretación epistémica. Si hemos de esperar algo de la filosofía política, nos interesan desarrollos que, al menos, nos permitan estar seguros de cuándo decir que no. Buscamos poder decir, con cierto criterio, que no todo vale igual. Pero no estoy muy convencido de que el no de todos sea un no relevante. A mi pa- 
recer, la comparación con la ciencia creo que se puede prolongar uno poco más. A pesar de Feyerabend y sus muchos herederos postmodernos, en ciencia - y sería de esperar en filosofía moral - hay un espacio por explorar entre el todo vale y lo único que vale es esto. Frente a un acontecimiento podemos disponer de diversas explicaciones todas ellas verdaderas, o, por mejor decir, más modestamente, compatibles con las observaciones. Es verdad que Kennedy murió porque le pegaron un tiro, pero también porque la bala —o las balas- entró por tal sitio y afectó a tal órgano, porque tenía el cráneo orientado en tal posición, y, vete tú a saber, porque el cirujano se puso la mar de nervioso o formaba parte de la conspiración, en fin, por un conjunto de condiciones individualmente necesarias y conjuntamente suficientes (la modesta condición INUSS, ya saben, tan inútil en otros negociados, como la atribución de responsabilidades, por eso, y acaso por dar gusto al gusto por los acrónimos, se inventan otras, como la NESS), que, además, se pueden ellas mismas desgranar hasta la microfísica. Es asunto complicado decir, sin más precisiones contextuales, que una explicación es más atendible que otra. Pero de lo que sí estamos seguros es de que no es verdad que a Kennedy lo atropello un camión. ¿Podemos esperar, en un sentido parecido, que nuestras teorías normativas nos permitan, por lo menos, un pesimista cabeceo orteguiano, un «no es eso, no es eso», frente a las acciones o situaciones políticas. Ernesto parece confiar en que sí, en que podemos confiar en hacerlo. El problema es si, por la vía de los consensos universales vamos muy allá, si el cabeceo no acaba por ser el delsesteo aburrido ante la vacuidad.

\section{EL LUGAR DE LA VIRTUD}

Llegado este punto, el problema es de siempre, el que nos impide seguir hasta el final con la comparación: en filosofía práctica no podemos acudir al auditor independiente del control empírico. Decir que esto es un serio problema sería añadir otra obviedad a las muchas que he venido hilvanado hasta aquí. Pero creo, y con esto recupero un cabo que dejé por rematar más arriba, que sí disponemos de otros tipos de controles, sin duda mucho más inseguros pero que algo nos pueden ayudar. Hay uno que Ernesto supera con la mejor nota: la veracidad en el trato con las propias ideas.

Como antes sugerí, en disciplinas con criterios de tasación inciertos, que uno se tome en serio las propias ideas, hasta el punto de que tenga que dejar su país o su posición social, es, al menos, una señal, de que tiene un trato honesto con ellas. Que no le pasa, vamos, como al otro MARX, recuerdan: «señora, si no le gustan mis principios, no se preocupe, tengo otros». Por supuesto, el compromiso veraz con las ideas no garantiza la verdad de las ideas, pero, eso no quita para que podamos dudar de las ideas de aquel que no asume las implicaciones prácticas que se siguen de ellas. Por eso descreemos del médico —y de sus conocimientos— que recomienda un tratamiento que él mismo no se aplica. Pero eso hay razones para prestar atención a aquellos que nos dicen cosas que no nos gusta oír, que no dicen cosas por afán de agradar. A falta de asideros más sólidos, que se tomen en serio a sí mismos es una invitación para tomar en serio lo que nos dicen. En filosofía práctica nunca está de más pedir algunas pruebas en primera persona de los talentos. Quizá no sea tan desatinada la opinión de que un filósofo infeliz —e incluso uno diría triste- es un mal filósofo, al menos a uno que viva en nuestra parte del mundo y sin un cupo excesivo de infortunios. 
Cuando no hay tribunal del mundo para medir la verdad, adquieren mayor importancia el amor a la verdad, el tribunal de la conciencia, el coraje intelectual, las virtudes epistémicas, como queramos llamarlo. En los cien metros lisos, con tribunales seguros, es fácil deslindar el trigo de la paja: el más rápido es el que llega el primero. Justicia procesal perfecta, que diría RAWLS. En física, casi, casi. Las cosas resultan más complicadas en buena parte de las ciencias sociales, por no mencionar el arte. Creo que en alguna parte FREUD dijo que la actividad intelectual es una cuestión de carácter. PAVESE lo expresó de un modo no sé si más inadecuado pero sin duda más rotundo: «No bastan las veleidades, las furias y los sueños; se necesita algo más: cojones duros». Se necesita porque no, no es fácil pensar a la intemperie, contra corriente. Quien seguro que lo dijo es otro filósofo, que no me resulta más simpático que el vienés, pero que en esto la cuadró. Como lo cito a través de Williams, confío que se disculpe que me acuerde de NIETZSCHE: «¿Pues, qué significa ser honesto en las cosas del espíritu? ¡Ser riguroso con el propio corazón, despreciar los bellos sentimientos, hacer de cada sí y de cada no un asunto de conciencia! [...] La "voluntad de verdad" no significa "no quiero dejarme engañar", sino — no queda otra alternativa— "no quiero engañar, ni aún a mí mismo": y con esto nos encontramos en el terreno de la moral».

WiLLIAMS incluso se anima y ordena el escalafón en un caso bien conocido: «Durante bastantes años, las gentes bienpensantes de la izquierda intelectual siguieron a SARTRE en su cruda marginación de CAMUS, en su desdén hacia lo que se presentaba como el necio humanismo, el moralismo subjetivo y la incompetencia filosófica de CAMUS. Puede que CAMUs haya sido un filósofo menos profesional que SARTRE, pero no está claro que fuera peor filósofo. Lo que con toda seguridad es cierto es que fue un hombre más honesto y su autoridad intelectual descansa en ese hecho». Un cuadro que WILLIAMS podría haber completado acordándose de cómo años más tarde otro mandarín parisino, macerado en una atmósfera intelectual no menos enrarecida, ALTHUSSER, poco antes de trastornarse irreparablemente, acabó por autocalificarse como fraude.

No creo yo que sea buena idea empezar a votar para ordenar la jerarquía de honestidades del gremio. Además, no nos engañemos, los resultados estarían trucados: siempre ganaría WiTTGENSTEIN; los suyos le votarían como una sola voz. Dejémoslo aquí. Mi interés por estos aspectos es tangencial, porque nos ayudan a iluminar lo que se espera del propio trabajo. Pero, en fin, si en uno de los muchos ratos de ocio que permite la profesión nos dejamos vencer por la tentación, estoy convencido que Ernesto andaría entre los mejores, en una liga bien distinta, a años luz, de aquellos que, lo admitan o no, podrían reconocerse en los versos de un poeta que Ernesto aprecia casi tanto como yo, cuando escribía: «la vocación, aquello sobre lo que fundamos un día nuestro ser, el nombre que le dimos a nuestra dignidad, vemos que no es más que un desolador deseo de esconderse».

\section{REFERENCIAS}

ARISTÓteles, 1985: Etica Nicomáquea. Etica Eudemia, Madrid: Gredos.

GARZÓN VALDÉS, E., 1989: «Ideología y humanismo en la obra de Francisco Miró Quesada», en Garzón VAldés, E., 1993: Derecho, ética y política, Madrid: Centro de Estudios Constitucionales. 
- 1998: «Razonabilidad y corrección moral», Claves de razón práctica, 88.

— 1998: «Algunas reflexiones sobre la ignorancia», en GARZÓN VALDÉs, E., 2001: Filosofía, política, Derecho, Universitat de Valencia.

Nussbaum, M.: «Lawyer for Humanity: Theory and Practice in Ancient Political Thought», en ShapIRO, I., y DeCEW, J. W. (edis.), 1995: Theory and Practice, New York: Nomos XXXVII, New York University Press.

Waldron, J.: «What Plato Would Allow», en Shapiro, I., y DeCEW, J. W. (edis.), 1995: Theory and Practice, New York: Nomos XXXVII, New York University Press.

Williams, B., 2002: Verdad y veracidad, Barcelona: Tusquets. 